\title{
MANUAL OPERATIVO PARA LA ELABORACIÓN DE «DEFINICIONES CONTEXTUALES» Y «REDES CONTRASTANTES»
}

\author{
Juan A. Magariños de Morentín
}

Universidad Nacional de La Plata (Argentina)

\section{INTRODUCCIÓN}

El mayor desafío al encarar un proyecto de investigación es encontrar la metodología adecuada a su objetivo. Durante varios años hemos ido elaborando nuestras pautas metodológicas, en relación con nuestro proyecto sobre el Análisis Semiótico del Discurso Político, cuyo encuadre actual tiene por título "¿Cuál es el futuro de la democracia en América Latina?». Una primera línea metodológica la desarrollamos en "La semiótica de enunciados», que actualmente forma parte de "Los fundamentos lógicos de la semiótica y su práctica» (Magariños de Morentín, 1996). Pero la aplicación de ese texto mostró que se hacían necesarias algunas explicaciones acerca de la operatoria concreta que permitiera utilizar plenamente la metodología que se proponía a los discursos en estudio. Una afortunada coyuntura nos exigió una respuesta más práctica a tales aspectos. La Organización Panamericana de la Salud (OPS), Delegación Argentina, solicitó nuestro asesora- 
miento metodológico, así como el concreto apoyo analítico de dos investigadoras del equipo: Teresa Poccioni y Nacy Fernández, para su Proyecto «Equidad de género en la dimensión socio-emocional de la calidad de atención en salud», que, con la dirección de Patricia Pittman, se desarrolla en la Argentina. De esta exigencia surgió este "Manual Operativo». Sus ejemplos están tomados de entrevistas en el ámbito de la salud, en sectores urbano-marginales; no obstante, metodológicamente, el tratamiento es homólogo al que se aplica al análisis del discurso político, ya bien sea sobre el texto de efectivos discursos de políticos, o sobre documentos, como lo hacemos actualmente al analizar la Declaración de Viña del Mar, surgida de la VI Cumbre Iberoamericana de Jefes de Estado y Presidentes de Gobierno, que tuvo lugar en noviembre de 1996, o sobre los artículos de periodistas políticos o sobre entrevistas a la comunidad para buscar el contraste, en el uso cotidiano, de los conceptos utilizados en los estamentos específicamente políticos. El método transciende la concreta tarea del análisis político y se propone como un instrumento importante, pero que nunca será el definitivo, para la comprensión de cómo se producen los contenidos de la significación en el ámbito de las ciencias sociales.

\section{SUPUESTOS Y DISCIPLINAS}

Las operaciones metodológicas que aquí se proponen y describen provienen de una síntesis entre la semiótica cognitiva y el análisis del discurso. Se basan en considerar que las investigaciones sociales son investigaciones científicas y que el objeto de conocimiento de tales investigaciones son los discursos sociales mediante los que determinada comunidad se representa/interpreta los fenómenos de su entorno. Por ello, la calidad del análisis acerca de tales discursos será la de constituir un metalenguaje ${ }^{1}$, específico a esos mismos discursos, consistiendo su valor científico en la identificación de las reglas mediante las que, en el caso específico del corpus en estudio, producen la representación/interpretación de los fenómenos de los que hablan.

1 Así quedó afirmado en otro trabajo (Magariños de Morentín, 1996: 271): «"En la medida en que toda proposición metalingüística relativa a los lenguajes naturales es analítica" (Lyons, 1977: 292), el carácter de las operaciones (analíticas) constitutivas de una metodología en ciencias sociales es metalinguiística». 
La semiótica cognitiva aporta la formulación de tres supuestos fundamentales: 1) no hay semántica ${ }^{2}$ sin sintaxis ${ }^{3}$ (lo que no implica afirmar la equivalencia entre una y otra); 2) todo lo efectivamente dicho se corresponde con una posibilidad de decirlo preexistente (esto equivale a decir que todo texto proviene de un sistema pertinente, el cual, al menos desde un punto de vista lógico, antecede a dicho texto), por lo que, a partir de lo efectivamente dicho puede inferirse el sistema sintáctico-semántico de donde procede, y 3) que estas posibilidades de decir no son individuales, sino que se comparten con la comunidad a la que pertence dicho productor del texto (en cuanto el productor de cualquier texto comparte alguna, al menos, o, por lo general, varias de las «formaciones discursivas» 4 vigentes en tal comunidad). La recuperación de estas «formaciones discursivas» es uno de los objetivos principales de la metodología semiótica que aquí se propone.

El análisis del discurso, por su parte, es una metodología cualitativa cuyo objetivo consiste en establecer el contenido semántico de los conceptos correspondiente a los términos efectivamente utilizados en determinados textos, cuyo análisis se considera interesante. Proviene, por una parte. del estructuralismo norteamericano, a través de los continuadores de la obra de Zellig Harris (1954), y, por otra, de la escuela francesa de análisis de discurso, originada en los trabajos linguiísticos de Michel Pêcheux $(1969,1975)$ y en las reflexiones sobre epistemología de la historia y crítica del discurso de Michel Foucault $(1969,1971)$, todo ello continuado por lingüistas, sociólogos y politicólogos vinculados a la revista Langages. En sus aspectos más actuales se basa en los desarrollos de la linguíistica cognitiva realizados, entre otros, por Ray Jackendoff (1983, 1987, 1993), Ronald Langacker (1987, 1991), George Lakoff \& Mark Johnson (1980) y George Lakoff (1987).

2 Se entiende aquí por semántica el estudio del significado, con toda la problemática que trae consigo el término «significado» en cuanto a las relaciones entre las palabras y las cosas o entre las palabras y determinadas entidades teóricas (por ejemplo, los «designata» de Carnap (Lyons, 1977: 117ss).

3 Se entiende aquí por sintaxis (de modo muy semejante a como lo hace Lyons, 1977: 375) un conjunto de reglas que da cuenta de la distribución de lexemas a través de las oraciones de la lengua, en cuanto tales reglas establecen sus combinaciones permisibles.

4 Podrá decirse que se ha identificado una formación discursiva (de modo muy semejante a como lo hace Foucault, 1969: 53) cuando, entre determinada cantidad de enunciados, identificables en un corpus de discursos producidos en determinada comunidad, pueda establecerse una regularidad en cuanto a la construcción de los objetos, de los tipos de enunciacion, de los conceptos y de las elecciones temáticas. 
Fundamentalmente, se diferencia del análisis del contenido ${ }^{5}$ al no admitir conocimiento a priori de ninguna clase, en cuanto al contenido semántico del lenguaje, sino que se propone explicar, respecto de cada término, de qué modo construye tal contenido o significación en función de su uso en el contexto material y positivo en el que aparece. El significado es una construcción cuya materia prima es lo efectivamente dicho en el discurso, sin que sea lícito acudir al conocimiento que pueda tenerse de la historia de las ideas o de la cultura de determinada comunidad (salvo que se aporten los textos correspondientes).

\section{OPERACIONES ANALÍTICAS}

Los que siguen son algunos criterios para la adopción de determinadas decisiones que debe tomar el analista al trabajar en el marco de $L a$ Semiótica de Enunciados (Magariños de Morentín y otros, 1993; Magariños de Morentín, J. A., 1996). Provienen de la experiencia analítica de investigaciones efectivamente realizadas durante los últimos 7 años. Se supone que se trabaja sobre textos originariamente producidos en forma escrita o ya bien sobre desgrabaciones escritas de textos originariamente producidos en forma oral.

Desde la perspectiva de La Semiótica de Enunciados, la primera operación analítica de intervención en un texto es la de segmentación. No obstante, por lo general, y especialmente tratándose de textos (desgrabaciones) procedentes de la oralidad, es necesario proceder a cierta normalización previa, en los estrictos límites y con las estrictas condiciones que se describen a continuación.

\subsection{La normalización}

Es tarea que exige un particular cuidado, para evitar (o advertir, cuando corresponda) el añadido o la eliminación, en el texto en estu-

5 La hipótesis directiva del análisis de contenido afirma la existencia, en el texto o en un corpus de textos, de indicadores (operativamente, «palabras clave»), con carácter de categorías interpretativas a priori, mediante cuya identificación se alcanza una caracterización contrastada de los productores de tales textos (Gardin, 1987: 55). 
dio, de términos o expresiones consideradas respectivamente procedentes o improcedentes de acuerdo con la interpretación (semántica) que realice el analista.

Tampoco se trata de una normalización que implique transformar un texto, adecuándolo a determinado modelo procedente de alguna gramática normativa, para establecer su forma «correcta». Con esta salvedad, la normalización puede requerir dos clases distintas de intervención: 1) recuperaciones, que predominantemente lo serán de correspondencias anafóricas, catafóricas o de implícitos sintácticos (sujetos elididos u otros recursos de la economía del habla); y 2) procesamiento de construcciones sintácticas incompletas (expresiones truncadas, cambios de estructuras gramaticales, etc.).

En lo que se refiere a la normalización, la intervención del analista puede ser estrictamente sintáctica o contener alguna inferencia semántica que, no obstante tener una base sintáctica, implique una apreciación que ubique en el texto un término o expresión cuya efectiva necesariedad sintáctica corre por riesgo y cuenta del analista. Ambas situaciones deben diferenciarse mediante las marcas gráficas metalinguísticas correspondientes. Se sugieren las siguientes: las incorporaciones sintácticamente justificadas se incluirán entre paréntesis: «(...)»; las incorporaciones de términos o expresiones que el analista considera que faltan por economía u omisión involuntaria del productor del texto, pero que no encuentran una efectiva justificación sintáctica, se incluirán entre corchetes: «[...]».

La importancia de marcar esta diferencia radica en dejar constancia del mayor margen de criticabilidad que ofrecen las segundas, frente a la mayor evidencia de efectiva probabilidad sintáctica de las primeras. En ningún caso, ante fenómenos semióticos: linguiísticos, gráficos, etc., se mueve el analista en los espacios de la necesariedad, ni puede permitirse el de la arbitrariedad absolutas; pero, como se pretende el máximo rigor y criticabilidad de los pasos analíticos por los que procede hasta llegar a las conclusiones que formule, debe dejar constancia de la calidad, más ceñida a la textualidad o más dependiente de alguna forma de interpretación, de las modificaciones que introduzca en el texto original. El lector que evalúe el proceso analítico debe estar en condiciones de aceptar o rechazar tales intervenciones modificatorias en cada uno de los pasos del análisis efectuado, ya a partir de esta inicial normalización. En definitiva, la normalización sólo es permisible en los casos en que lo requiera la comprensiblidad elemental del texto que se analiza, lo que, ante cada segmento hacia cuya identifica- 
ción se tiende, implica completar la estructura sintáctica de la expresión en estudio. (Ver ANEXO: I. NORMALIZACIÓN).

\subsection{La segmentación}

En cuanto a la segmentación, ésta tiene por objetivo disponer de las partes de un texto que se consideran básicas o elementales (no mínimas, en el preciso sentido semántico, ya que eso lo constituyen los «enunciados»; ver Magariños de Morentín y otros, 1993: 9) para la construcción de la significación. El criterio intuitivo e inmediato (que excluimos) para establecer los cortes inicial y final de cada segmento se apoyaría en la idea de la producción de una unidad semántica. El riesgo de seguir este criterio consiste en la subjetividad inherente a la identificación de determinada secuencia textual como la unidad semántica en cuestión. Dado que todo segmento está incluido en una unidad semántica mayor, con la totalidad de la cual guarda, supuestamente, relaciones de contigüidad, continuidad, coherencia y cohesión semánticas, establecer el comienzo y el final del segmento en estudio podría abarcar dimensiones que perjudicarían la tarea analítica, ya que dependerían, caso de adoptarse el criterio semántico, de decisiones individuales del analista, no siempre (o más bien, en pocas oportunidades) intersubjetivamente compartibles.

Esto hace que la adopación de un criterio sintáctico proporcione un fundamento más objetivo, aunque tampoco exento de problemas. La sintaxis (para cuya identificación puede seguirse alguna de las gramáticas de vigencia reconocida) garantiza la objetividad por pertenecer a una disciplina diferente a aquella o aquellas involucradas en la investigación (salvo en el caso de un análisis metalinguístico, lo que, provisionalmente, excluimos); el recorte, por tanto, no estará influido por los preconceptos que el manejo de las correspondientes disciplinas puede inducir en el analista. Esto no contradice lo afirmado anteriormente acerca del rechazo a tomar como modelo alguna gramática normativa para establecer la forma correcta de determinado texto; no es lo mismo analizar la presencia y la eficacia de determinadas categorías gramaticales en función de alguna determinada sintaxis, que es lo que aquí se propone, que corregir estructuras oracionales supuestamente mal formadas según alguna gramática de un hablante-oyente ideal, que es lo que aquí se excluye. 
El criterio sintáctico cuenta, en principio, con el aval, casi unánime, de los estudiosos de la semántica, en cuanto consideran la sintaxis como el conjunto de las relaciones necesarias, pero no suficientes, para la construcción del significado (Jackendoff, 1983: 57). Aplicar el criterio sintáctico para identificar los segmentos textuales elementales con valor semántico implica dejar provisionalmente de lado la subjetividad del analista y contar con la aceptación, por parte de este analista, de la eficacia de una operación para la cual dicho analista se somete a reglas objetivas, formalmente enunciadas e independientes de la interpretación que él pueda formular respecto del texto que analiza.

El criterio sintáctico de segmentación puede formularse en los siguientes términos: se marcará el comienzo y el final de un proceso textual (en sentido hjelmsleviano) tal que contenga una oración de base y sus modalizadores (si los hubiese). Se entiende por «oración de base» el mínimo textual que puede quedar representado por la proforma: $F N+F V[F V=V+F N]$.

A esta oración de base se le incorporarán las modalizaciones que afecten a cualquiera y a todos sus componentes sintácticos. Esto quiere decir que, a partir de una estructura (si está completa) del tipo: FN + [V + FN], se identificarán y se incorporarán, como partes integrantes de la segmentación buscada, todos aquellos términos y/o expresiones que aparezcan en vinculación sintáctica con cualquiera de las tres partes (posibles) de la oración de base. Al exigir que tales modalizaciones se encuentren sintácticamente vinculadas, se excluyen las vinculaciones que puedan establecerse con otras partes del texto en virtud de relaciones puramente semánticas. $O$ sea, de cada modalización aceptada como sintácticamente vinculada a alguno de los componentes de una oración de base, debe poderse identificar la marca textual que establece dicha vinculación.

Dependiendo de lo que el analista busca establecer (en cuanto al sistema virtual de tales posibilidades) pueden tomarse todos los segmentos identificados o seleccionar aquellos que son pertinentes para la finalidad del análisis en cuestión. Aquí otro punto de riesgo, ya que la selección puede sesgar el análisis hacia una evaluación preconcebida. En este sentido, la exigencia de exhaustividad planteada ya por J.-J. Courtine (1981:22) requiere tomar en consideración cada uno de tales segmentos; incluso, para excluirlos, deberá establecerse la razón de esa exclusión. (Ver ANEXO: II. SEGMENTACIÓN). 


\subsection{Las definiciones contextuales}

Supongamos, por ejemplo, que lo que se trata de establecer, según nuestro texto, son las características de la construcción semántica de determinados nombres, en función de los contextos en que los ha utilizado su productor; tal es la finalidad fundamental de las llamadas «definiciones contextuales», hacia las que se dirige la continuación de la tarea analítica.

Es muy posible que, en cada caso, haya que efectuar, todavía, determinadas modificaciones, que se irán viendo. Resulta imposible prever y categorizar, sistematizándolas, todas las situaciones que puedan presentarse, ya que, por tratarse en este caso de un registro de la oralidad, entramos en aquel universo «heteróclito» del que hablaba Saussure, más allá de lo que los estudios pragmáticos han podido normalizar.

La definición contextual es quella mediante la cual se establece el sentido que adquiere un término cualquiera, presente en determinado segmento textual completo ${ }^{6}$, en función del contexto al que dicho término aparece asociado en ese mismo segmento.

Con mayor precisión, también puede decirse que la definición contextual consiste en la transformación de determinado segmento textual completo, de modo tal que determinado nombre, efectivamente utilizado en dicho segmento textual, ocupe el lugar del $\mathrm{N}$ de la FN de una oración copulativa con cláusula de relativo ${ }^{7}$ (según alguna de las variantes de «es aquél que...»), constituyendo el V («es») junto con dicha cláusula («aquél que...») la FV que completa la definición contextual.

La definición contextual tendrá una configuración semejante a la siguiente:

$\mathrm{X}$ es [aquel/la/lo + (preposición o expresión preposicional con q! ${ }^{8}$ ) + (resto del contexto de la oración)].

6 En esta definición, se entiende por «segmento textual completo» al constituido (o reconstituido, según la tarea de normalización) por una oración de base ([FN + FV] o $\left[\mathrm{FN}_{1}+\mathrm{V}+\mathrm{FN}_{2}\right]$ ) y los modalizadores de cada uno de sus componentes, si los hubiera en el texto que se analiza.

7 En esta definición, «la oración copulativa con cláusula de relativo» estará constituida por el resto del segmento en análisis, en cuanto contexto de dicho nombre, con las transformaciones en la estructura sintáctica que exija la modificación operada.

8 Se entiende por «q!» cualquiera de aquellas expresiones sintácticas que contienen un pronombre relativo expresado directamente o por transformación (en el sentido en 
En cuanto a la «preposición o expresión preposicional», en función de la cual se rearticulará sintácticamente el resto del segmento textual completo, ella proporcionará expresiones sintácticas del tipo (en cada caso exigidas por la estructura sintáctica del texto original):

$\mathrm{X}$ es [aquel/la/lo + que + (resto del contexto de la oración)]

$\mathrm{X}$ es [aquel/la/lo + en el que + (resto del contexto de la oración)]

$\mathrm{X}$ es [aquel/la/lo + con el que + (resto del contexto de la oración)]

$\mathrm{X}$ es [aquel/la/lo + para el que + (resto del contexto de la oración)]

$\mathrm{X}$ es [aquel/la/lo + desde el cual + (resto del contexto de la oración)]

$\mathrm{X}$ es [aquel/1a/lo + respecto del cual + (resto del contexto de la oración)]

$\mathrm{X}$ es [aquel/la/lo + cuyo + (resto del contexto de la oración)] etc.

siendo esta enumeración meramente ejemplificativa. (VER ANEXO: III. DEFINICIONES CONTEXTUALES).

\subsection{Ejes conceptuales, redes secuenciales y contrastativas}

El siguiente paso, a partir de un determinado repertorio de definiciones contextuales, consiste en identificar los ejes conceptuales ordenadores, según los cuales pueden agruparse las definiciones obtenidas. Los conjuntos así constituidos son representativos de los distintos modos de atribuir significado a los correspondientes términos por parte de la comunidad o sector social que produjo los discursos en estudio.

Toda definición contextual genera un eje conceptual que permite realizar búsquedas (preferentemente, a partir de un banco de datos nutrido con el conjunto de las definiciones analíticamente obtenidas) mediante las que se nucleen otras definiciones que comparten el mismo eje. (Ver ANEXO: IV. EJES Y REDES). 
Una de las características del método es que permite identificar los ejes según los cuales distintos sectores de la comunidad le confieren distinto significado a los mismos términos, según lo que ha sido efectivamente dicho, así como también permite identificar las coincidencias. Si bien se puede estudiar de qué modo un determinado modelo construido a priori se encuentra efectivamente utilizado en el discurso de una comunidad (lo que caracteriza al método estructuralista y algunas variantes del análisis del contenido), la principal utilidad diferencial del método que aquí se propone es descubrir cuáles son las componentes y/o los rasgos prototípicos de determinados conceptos en estudio, tal como han sido utilizados en determinado momento por dicha comunidad (lo que caracteriza a esta práctica de la semiótica cognitiva y del análisis del discurso).

El análisis puede continuar estableciendo sub-ejes en función de la profundidad de los objetivos que tenga la investigación. Una nueva lectura de los sub-ejes encontrados en cada eje permite reconstruir el correspondiente árbol.

En la medida en que tales árboles o redes se extraigan de uno o de varios discursos, darán lugar a una Red Secuencial o a una Red Contrastativa. Un análisis no estará adecuadamente concluido hasta que, en el seno de una Red Contrastativa, no aparezcan diferencias o contradicciones que garanticen que se ha llegado a la identificación de, al menos, más de una Formación Discursiva. La presencia, pues, de la diferencia o de la contradicción —el contenido de tal «diferencia o contradicción» deberá ser tal que provoque la inconsistencia del sistema axiológico y/o conceptual en estudio (Magariños de Morentín, 1996: 434) - es condición necesaria pero no suficiente para afirmar la representatividad de la muestra sometida a análisis. Esta representatividad no tiene un fundamento estadístico, sino constructivo: debe acreditar que está representada la pluralidad social, de la que se afirma como hipótesis básica que es inherente a toda comunidad (lo que se confirma en la medida en que toda investigación demuestra la inconsistencia del sistema cultural de cualquier comunidad en estudio).

Todo lo cual va configurando el universo de valores y conceptos vigentes en la comunidad a la que pertenece quien habla.

La representación de la configuración diferencial de dicho universo de valores y conceptos, tal como aparece efectivamente enunciado en determinado corpus de discursos, es el objetivo específico al que aporta sus operaciones analíticas esta metodología semiótica. 


\section{ANEXO}

Se presenta aquí un ejemplo tomado de una de las entrevistas realizadas para el estudio. Se trata de segmentar el siguiente texto a los efectos de proceder al análisis de sus características en cuanto productor de significaciones.

Pregunta: Y dígame, ¿cuál es su experiencia con los médicos?

Respuesta: Mirá, yo he ido a médicos clínicos que no saben un cuerno. ¿Sabés lo que pasa? Yo te explico. Vos cuando tenés una enfermedad, el médico ¿sabés lo que hace? Te dice: «Bueno, tiene que tomar esto», qué sé yo, «el régimen éste»; pero no te explica cómo tenés que..., por qué es eso, ¿entendés? Entonces, ¿vos tenés conciencia de lo que tenés?, ¿por qué?, ¿cuál es el problema de tu enfermedad?, ¿cómo apareció? Porque a mí me apareció de grande, en mi familia no hay ningún diabético y yo tengo muchachos que por ahí tienen síntomas de diabético y no lo saben y les digo: «pero, ihacete un coso!» $\mathrm{Y}$ ellos dicen: «Sí, porque tengo sed, orino mucho de noche, que patatín, patatán» y uno no le da bolilla a eso, ¿viste? De cada un diabético hay otro diabético, ¿sabías vos eso?

\section{NORMALIZACIÓN}

mirá\# ${ }^{9}$ yo he ido a médicos clínicos que no saben un cuerno\# $i(+v o s){ }^{10}$ sabés lo que pasa?\# yo te explico (=lo que pasa)\# vos cuan-

9 Los signos de puntuación, en la transcripción del discurso oral, constituyen una intervención del analista (o de quien realiza la desgrabación). Son diversos los criterios que pueden asumirse; en este trabajo se ha optado por sustituir el punto «.», la coma «,», el punto-y-coma «;» y los dos-puntos «:», por el signo «\#», para desemantizarlo de su sentido de acabamiento o de interrupción temporal en el desarrollo de una expresión y para que el signo diferente propuesto (\#) advierta al lector de que, en correspondencia con algún tipo de pausa realizada por el productor del texto, meramente se está proponiendo un corte cuya duración es materia de interpretación y que incluso podría eliminarse, si se considera que corresponde hacerlo. No obstante, el signo (\#) es importante ya que permite reflexionar sobre la estructura sintáctica que se está normalizando.

10 Según anticipamos, en este caso, como en los restantes que aparecen entre paréntesis «(...)», el texto recuperado implica una mínima intervención del analista ya que, ya bien se trata de un sujeto implícito (como ocurre en este concreto primer caso, en cuanto inferencia directa de la actuación pragmática; lo que se marca anteponiendo el 
do tenés una enfermedad\# el médico $九(+v o s)$ sabés lo que hace?\# $(+e l$ médico) te dice\# bueno\# (+usted) tiene que tomar esto [un remedio]\# qué sé yo [lo que (+el medico) te dice]\# (=el médico te dice (+usted) tiene que tomar) el régimen éste\# pero $(+e l$ médico) no te explica cómo (+vos) tenés que [cuidarte] ${ }^{11} \#((+e l$ médico $)$ no te explica) por qué es eso (=que tengás una enfermedad)\# i(+vos) entendés [lo que digo]?\# entonces\# ¿vos tenés conciencia de lo (=la enfermedad) que tenés?\# ¿(+vos tenés conciencia de) por qué [tenés la enfermedad]?\# ¿(+vos tenés conciencia de) cuál es el problema de tu enfermedad?\# ¿(+vos tenés conciencia de) cómo apareció (+tu enfermedad)?\# porque a mí (+la enfermedad) me apareció de grande\# en mi familia no hay ningún diabético\# y yo tengo muchachos [conocidos] que por ahí tienen síntomas de diabético y (+los muchachos) no lo (=que tienen síntomas de diabético) saben\# y (a los muchachos=) les digo\# pero\# ¡hacete un coso! [análisis]\# y ellos dicen\# (me hago un análisis=) sí\# porque tengo sed\# ( $+y$ (los muchachos=) ellos dicen\# (me hago un análisis=) sí\# porque) orino mucho de noche\# (+y (los muchachos=) ellos dicen) que patatín, patatán\# y uno no le da bolilla a eso $\left(={ }^{¿}\right.$ viste?\# de [*por*] ${ }^{12}$ cada un diabético [conocido] hay otro diabético [desconocido]) \# ¿viste?\# de [*por*] cada un diabético [conocido] hay otro diabético [desconocido] ¿sabías vos (que de [*por*] cada un diabético [conocido] hay otro diabético [desconocido]=) eso?

signo «+» a dicho sujeto implícito) o ya bien se trata de la recuperación anafórica de un término o expresión que ya ha sido efectivamente dicha (en cuyo caso se ubica el paréntesis antes del correspondiente pronombre y el signo «=» dentro y fin derecho del paréntesis) o de la recuperación catafórica de un término o expresión que aparecerá en la continuación inmediata o relativamente próxima del texto (en cuyo caso se ubica el paréntesis después del pronombre, y el signo «=» dentro y al comienzo izquierdo del paréntesis); no obstante, siempre es una zona de riesgo.

${ }_{1}$ Es muy posible que «cuidarte» sea el verbo que el productor del texto no ha llegado a enunciar, pero no hay ninguna marca que así lo exija o lo justifique; sólo existe una ausencia por la incompletez de la sintaxis y una intuicion del analista, en cuanto hablante nativo, por captación de la isotopía que viene generándose en la respuesta. Puede decirse que el analista corre un mayor riesgo al incorporar esta expresión que en los casos comentados sub 2. Por ello, conforme a lo dicho anteriormente, el analista ubicará su incrustación entre conchetes «[...]», preservando así la calidad de criticable que corresponde a cada una de las intervenciones que lleva a cabo sobre el texto en estudio.

${ }_{12}$ Cuando, para salvar el sentido, la incorporación implica, además, una corrección gramatical respecto de la forma utilizada por el productor del texto (y el analista se decide a yuxtaponer tal corrección junto a la expresión originaria), ello se marca, en este trabajo, incluyendo el agregado corrector entre corchetes y asteriscos: $\ll[* \ldots *] »$. 


\section{SEGMENTACIÓN}

Supongamos completada la normalización y procedamos a la segmentación del mismo texto; en el anterior texto normalizado, se identifican los siguientes segmentos:

\section{1. mirá}

2. yo he ido a médicos clínicos que no saben un cuerno

3. ¿(+vos) sabés lo que pasa?

4. yo te explico (=lo que pasa)

5. vos cuando tenés una enfermedad el médico ¿(+vos) sabés lo que hace?

6. (+el médico) te dice bueno (+usted) tiene que tomar esto [un remedio]

7. qué sé yo [lo que (+el medico) te dice]

8. $(=(+e l$ médico) te dice $(+$ usted) tiene que tomar) el régimen éste

9. pero (+el médico) no te explica cómo (+vos) tenés que Icuidarte]

10. ((+el médico) no te explica) por qué es eso (=que tengás una enfermedad)

11. ¿(+vos) entendés [lo que digo]?

12. entonces ¿vos tenés conciencia de lo (=la enfermedad) que tenés?

13. ¿(+vos tenés conciencia de) por qué [tenés la enfermedad]?

14. ¿(+vos tenés conciencia de) ¿cuál es el problema de tu enfermedad?

15. ¿ (+vos tenés conciencia de) cómo apareció (+tu enfermedad)?

16. porque a mí (+la enfermedad) me apareció de grande

17. en mi familia no hay ningún diabético

18. y yo tengo muchachos [conocidos] que por ahí tienen síntomas de diabético 
19. y (+los muchachos) no lo (=que tienen síntomas de diabético) saben

20. y (a los muchachos $=$ ) les digo pero ¡hacete un coso! [análisis]

21. y (los muchachos=) ellos dicen (me hago un análisis=) sí porque tengo sed

22. (+y (los muchachos=) ellos dicen\# (me hago un análisis=) sí\# porque) orino mucho de noche

23. (+y (los muchachos $=)$ ellos dicen) que patatín, patatán

24. y uno no le da bolilla a eso (=que de [*por*]cada un diabético [conocido] hay otro diabético [desconocido])

25. ¿viste?

26. de $\left[{ }^{*}\right.$ por*] cada un diabético [conocido] hay otro diabético [desconocido]

27. ¿sabías vos (que de cada un diabético [conocido] hay otro diabético [desconocido]=) eso?

Estos 27 segmentos son los que permiten recuperar las distintas significaciones de que disponía previamente el productor del texto y que ha actualizado en él al producirlo. Esta recuperación se cumple mediante la elaboración de las definiciones contextuales inherentes al texto que se analiza.

\section{DEFINICIONES CONTEXTUALES}

Se puede, en principio, prescindir de determinados segmentos cuya función se hipotetiza como fundamentalmente «fática», en el sentido en que usa este término Jakobson (1963: 28ss). Tales segmentos serían los siguientes:

1. mirá

3. ¿(+vos) sabés lo que pasa? 
4. yo te explico (=lo que pasa)

7. qué sé yo [lo que (+el medico) te dice]

11. ¿(+vos) entendés [lo que digo]?

25. ¿viste?

Con el resto se puede proceder a elaborar las definiciones contextuales en las que el analista esté interesado.

Retomando el análisis del párrafo inicial, las siguientes son definiciones contextuales que proceden de los segmentos no excluidos anteriormente.

2. MÉDICOS son aquellos clínicos a los que yo he ido que no saben un cuerno

5. MÉDICO es aquel que ¿(+vos) sabés lo que hace cuando vos tenes una enfermedad?

6. (MÉDICO) es aquel que te dice bueno (+usted) tiene que tomar esto [un remedio]

8. (MÉDICO) (aquel que te dice (+usted) tiene que tomar) el régimen éste

9. /pero/ (MÉDICO) es aquel que no te explica cómo (+vos) tenés que [cuidarte] (8)

10. (MÉDICO) es aquel que no te explica) por qué es eso (=que tengás una enfermedad)

12. /entonces/ (ENFERMEDAD) es aquella que de que la tenés ¿vos tenés conciencia?

13. [ENFERMEDAD] es aquella que de por qué la tenés $i(+v o s$ tenés conciencia)?

14. ENFERMEDAD es aquella acerca de cuyo problema i(+vos tenés conciencia de) cuál es?

15. (ENFERMEDAD) es aquella tuya acerca de la cual $i(+v o s$ tenés conciencia de) cómo apareció ?

16. /porque/ (ENFERMEDAD) es aquella que a mí me apareció de grande 
17. DIABÉTICO es aquel del que no hay ninguno en mi familia

18. /y/ DIABÉTICO es aquel cuyos síntomas yo tengo muchachos [conocidos] que por ahí los tienen

19. (DIABÉTICO) es aquel cuyos síntomas tienen $(+$ los muchachos) y no lo saben

20. /y/ (MUCHACHOS) son aquellos a los que les digo pero ihacete un coso! [análisis]

21. /y/ (MUCHACHOS) son aquellos que dicen (me hago un análisis=) sí porque tengo sed

22. /y/ (MUCHACHOS) son aquellos que dicen (me hago un análisis=) sï\# porque) orino mucho de noche

23. /y/ ((MUCHACHOS) son aquellos que dicen) que patatín, patatán

24. /y/ ESO (que de [*por*] cada un diabético [conocido] hay otro diabético [desconocido]) es aquello a lo que uno no le da bolilla

26. DIABÉTICO es aquel [conocido] de $\left[{ }^{*} p o{ }^{*}\right]$ cada uno de los cuales hay otro diabético [desconocido]

27. ESO (que de [*por*] cada un diabético [conocido] hay otro diabético [desconocido]=) es aquello que ¿vos sabías?

Se hace evidente, a partir de esta última tarea de construcción de definiciones contextuales, que se está obteniendo un «diccionario» con los valores semánticos que el productor del texto le confiere a los términos que usa en su discurso (podían ser otros cualesquiera de los sustantivos efectivamente utilizados).

Aquí, por ejemplo, «MÉDICO» se presenta construido semánticamente del siguiente modo:

\section{«MÉDICO»}

2-aquellos clínicos a los que yo he ido que no saben un cuerno

5-aquel que $\measuredangle(+v o s)$ sabés lo que hace cuando vos tenes una enfermedad?

6-aquel que te dice bueno $(+u s t e d)$ tiene que tomar esto [un remedio] 
8-(aquel que te dice (+usted) tiene que tomar) el régimen éste

9-aquel que no te explica cómo (+vos) tenés que [cuidarte]

10-(aquel que no te explica) por qué es eso (=que tengás una enfermedad)

También se define «ENFERMEDAD»:

12-aquella que de que la tenés ¿vos tenés conciencia?

13-aquella que de por qué la tenés ¿(+vos tenés conciencia)?

14-aquella tuya acerca de cuyo problema ¿(+vos tenés conciencia de) cómo apareció ?

15-aquella tuya acerca de la cual i(+vos tenés conciencia de) cómo apareció ?

16-(enfermedad) es aquella que a mí me apareció de grande

También «DIABÉTICO»:

17-aquel del que no hay ninguno en mi familia

18-aquel cuyos síntomas yo tengo muchachos [conocidos] que por ahí los tienen

19-aquel cuyos sintomas tienen (+los muchachos) y no lo saben

26-aquel [conocido] de ${ }^{*}$ por $\left.^{*}\right]$ cada uno de los cuales hay otro diabético [desconocido]

Se puede continuar con «MUCHACHOS»:

20-aquellos a los que les digo pero ¡hacete un coso! [análisis]

21-aquellos que dicen (me hago un análisis=) sí porque tengo sed

22-aquellos que dicen ((me hago un análisis=) sí porque) orino mucho de noche

23-aquellos (que dicen) que patatín, patatán 


\section{EJES Y REDES}

El fragmento que se ha tomado en consideración para extraer estas definiciones era breve; puede comprenderse fácilmente la información que este método permite obtener de entrevistas completas, cuya duración promedio puede estimarse en 45'.

Por ejemplo, los ejes encontrados en el conjunto de definiciones de «Médico» que acaba de transcribirse son los siguientes:

\section{«MÉDICO»}

\section{EJE 1: CONOCIMIENTO}

\section{2-aquellos clínicos a los que yo he ido que no saben un cuerno}

-el termino subrayado «saben» proporciona la base lexemática para agrupar todas aquellas definiciones que lo contengan en su contexto (definiciones que pueden ser sólo las del término «MEDICO», pero también la totalidad u otra parte de las definiciones registradas, en cada caso según el interés del analista)

-es posible que el lexema de base seleccionado (aquí «saben») no agote la construcción del significado de «MÉDICO» en su relación con el concepto de «CONOCIMIENTO»; en tal caso se construirá un campo semántico en que se asocien, además, otros lexemas o expresiones lexemáticas afines, como «informado» o «estar informado», «estudio» o «estudioso», «investiga» y el propio «conocimiento» o «conocer»; en tal caso, el investigador debe hacer explícito el repertorio lexemático que considera constitutivo de un «campo semántico» de importancia para su análisis; así dicho campo será completo y criticable, dos cualidades necesarias para que una investigación social sea adecuada y rigurosa

—estos comentarios son aplicables a los restantes ejemplos de ejes

\section{EJE 2: PRAXIS}

5-aquel que ¿(+vos) sabés lo que hace cuando vos tenes una enfermedad?

- si esta definición contextual, en una búsqueda en la base de datos (constituidos aquí por las definiciones contextuales) que se esté utilizando, viniese junto con la anterior, por compartir en su contexto el fragmento lexemático «sab» (no la raíz, sino la secuencia significante que es constante en todas las variaciones del término), sería eliminada del conjunto conceptual que se está construyendo, ya que no se refiere al saber del médi- 
co, sino, textualmente considerado, al del interlocutor (entrevistador) con el que habla el autor del texto; no puede pretenderse una aplicación automática y acrítica de las reglas; hay que tener presente que se trata de que: (a) los ejes surjan del texto y no de supuestos externos aportados por el analista y (b) el analista haga explícitos sus criterios de aceptación o exclusión —o sea, «hace» es el lexema según el cual se ha identificado el eje «PRAXIS»

\section{EJE 3: TRATAMIENTO} dio]

6-aquel que te dice bueno (+usted) tiene que tomar esto [un reme-

8-aquel que te dice (+usted) (tiene que tomar) el régimen éste

- sería interesante explorar el «tener que» vinculado a las definiciones contextuales de «MÉDICO»

-también puede construirse el eje «TRATAMIENTO» de acuerdo con los lexemas «remedio» 0 «régimen»

-también, dependiendo del objetivo de la investigación, este eje «TRATAMIENTO» puede considerarse como un sub-eje del eje «PRAXIS»

\section{EJE 4: COMUNICACIÓN}

9-aquel que no te explica cómo (+vos) tenés que [cuidarte]

10-(aquel que no te explica) por qué es eso (=que tengás una enfermedad)

Las calidades encontradas en el interior de las expresiones que han construido los precedentes ejes, permiten identificar sub-ejes que van enriqueciendo el contenido del concepto efectivamente utilizado en el texto y con las que se va construyendo determinada Formación Discursiva. 


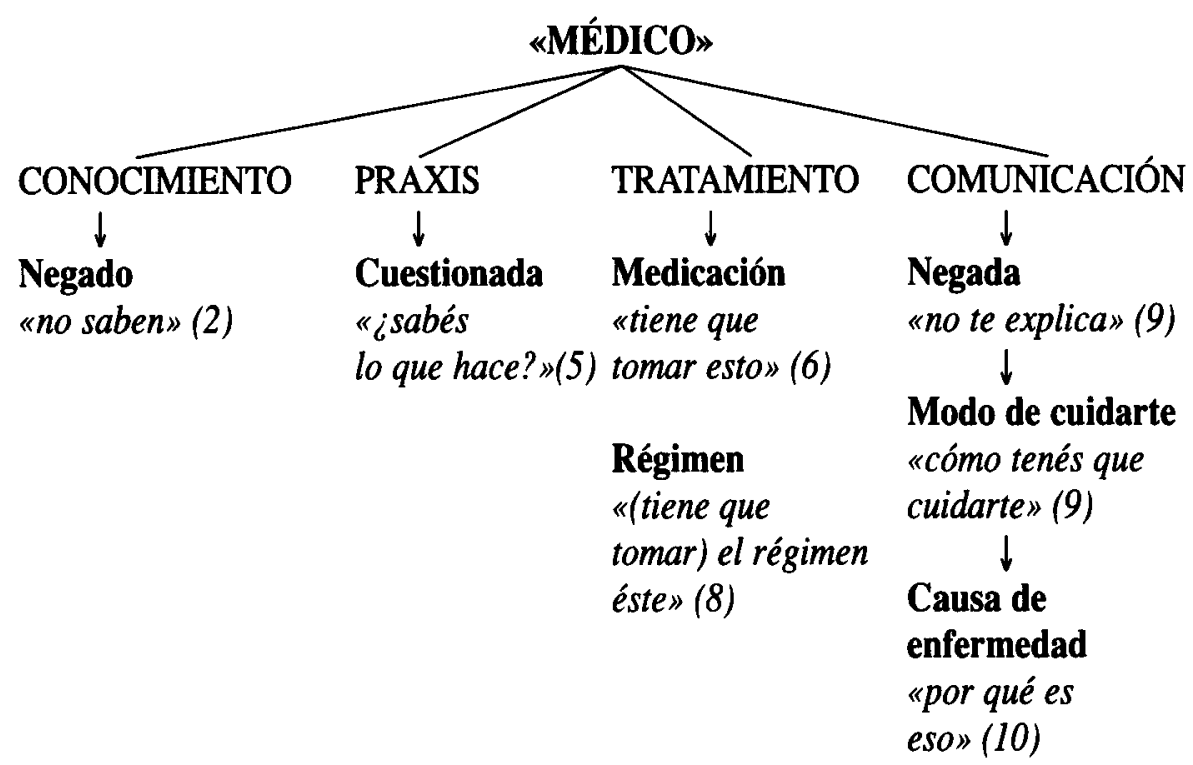

Suponiendo que este conjunto de ejes y sub-ejes tuviera la riqueza suficiente y necesaria para constituir una Formación Discursiva (en el ejemplo, meramente, uno de sus mínimos fragmentos), una variación en cualquiera de sus componentes tal que, caso de incluirla en la Formación Discursiva original, conduzca a afirmar y negar equivalentemente una misma y determinada proposición, estaría indicando la necesidad de originar la representación de otra Formación Discursiva. Esta (y otras que puedan identificarse) y la inicial constituirán el repertorio de Formaciones Discursivas disponibles en la comunidad en estudio, cada una de las cuales será internamente consistente, pero cuyo conjunto describiría las características específicas de la inconsistencia que, por hipótesis, se considera inherente a todo sistema cultural, y que el hallazgo correspondiente probaría.

\section{Referencias bibliográficas}

COURTINE, JEAN-JACQUES (1981). «Quelques problèmes théoriques et méthodologiques en analyse du discours». Langages 62, 9-127

FouCAULT, MiCHEL (1969). L'archéologie du savoir. París: Gallimard.

- (1971). L'ordre du discours. París: Gallimard. 
GARDIN, JEAN-Claude (1987). La logique du plausible. Essais d'épistéologie pratique en sciences humaines. París: Editions de La Maison des Sciences de L'Homme.

HARRIS, Zellig S. (1954). «Distributional Structure». Word 10, 146-162.

JACKendoff, RaY (1983). Semantics and Cognition. Cambridge: The MIT Press.

- (1987). Consciousness and the Computational Mind. Cambridge: The MIT Press.

- (1993). Languages of the Mind. Cambridge: The MIT Press.

JAKOBSON, ROMAN (1963). Essais de linguistique générale. París: Minuit.

LAKOFF, GEORGE (1987). Women, fire, and dangerous things: What categories reveal about the mind. Chicago: Chicago University Press.

LAKOFF, GEORGE \& MARK JOHNSON (1980). Metaphors we live by. Chicago: Chicago University Press.

LANGACKER, RoNALD (1987). Foundations of Cognitive Grammar. I: Theoretical Prerequisites. Stanford: Stanford University Press.

- (1991) Foundations of Cognitive Grammar. II: Descriptive Application. Stanford: Stanford University Press.

LyONS, JoHN (1977) Semantics. Volume 1 and 2. Cambridge: Cambridge University Press.

Magariños DE Moretín, Juan A. y otros (1993). La Semiótica de Enunciados. La Plata: IICS, Universidad Nacional de La Plata (incluido en 1996: 301-395).

- (1996). Los fundamentos lógicos de la semiótica y su práctica. Buenos Aires: Edicial.

PeCheuX, Mrchel (1969). Analyse automatique du discours. París: Dunod.

- (1975). Les vérités de La Palice. París: Maspéro. 